\title{
El modelo flipped classroom, una forma de promover la autorregulación y la metacognición en el desarrollo de la educación estadística
}

\author{
Cristina SÁNCHEZ-CRUZADO \\ M. a Teresa SÁNCHEZ-COMPAÑA
}

Datos de contacto:

Cristina Sánchez Cruzado Universidad de Málaga

cristinasanchez@uma.es

M. Teresa Sánchez Compaña Universidad de Málaga teresasanchez@uma.es

\begin{abstract}
RESUMEN
El objetivo de este estudio es obtener información a partir de una experiencia exploratoria, realizada en el Grado de Educación Primaria de la Universidad de Málaga, dentro del marco de una investigación-acción, en la que se utiliza el modelo flipped classroom en educación estadística, para evidenciar que, mediante la utilización de este modelo, se fomenta la autorregulación en el proceso de enseñanzaaprendizaje, alcanzando la metacognición. Se pretende, desde un planteamiento metodológico activo, basado en la autonomía personal y la autorregulación, implicar al alumnado en su aprendizaje, promover la capacidad de análisis de datos y razonamiento estadístico, y además ser capaces de desarrollar el pensamiento crítico. De acuerdo a los resultados observados, con este modelo se consiguen desplazar fuera del aula las actividades matemáticas más instrumentales, favoreciendo el desarrollo de tareas mucho más funcionales y formativas en el aula.
\end{abstract}

PALABRAS CLAVE: Flipped classroom; Metacognición; Autorregulación del aprendizaje; Educación estadística. 


\title{
The Flipped Classroom Model, a way to promote Self- Regulation and Metacognition in the Development of Statistical Education
}

\begin{abstract}
The objective of this study is to gain information taken from an exploratory experiment, which was carried out in an action-research framework, in the Primary Education Degree of the University of Malaga. In this experiment, the flipped classroom model is used in statistical education in order to show that self-regulation, in the teaching-learning process can be developed to the point of metacognition. It is intended to involve students in the learning process, promote their ability to analyse data and statistical reasoning, and allow them to develop critical thinking skills. It takes on an active methodological approach, and bases itself on personal autonomy and self-regulation. According to the results observed, the more instrumental mathematical activities can be left outside the classroom, thereby favouring the development of much more functional and formative tasks within the classroom.
\end{abstract}

KEYWORDS: Flipped classroom; Metacognition; Self-regulation learning; Statistical education.

\section{Introducción}

La estadística, es una rama de la matemática que aparece en el currículo de todos los niveles educativos, por su alto valor funcional en la vida personal y profesional. A pesar de ello, un gran número de estudiantes, acaban los cursos de estadística y no son realmente competentes para utilizar procedimientos estadísticos, y no entienden realmente qué significan los datos que manejan en un estudio (Shaughnessy, 2007).

La estadística, forma parte de una educación matemática que debe ser un proceso social, no exclusivamente instrumental. Esto es un hecho que suele sacrificarse, tomando mucho más peso la necesidad de lograr técnicas matemáticas, y conseguir una educación científicomatemática con una aplicación directa (Bishop, 1999).

Por otro lado, las tecnologías de la información y comunicación (TIC) aplicadas a los procesos educativos, han transformado la forma en la que se aprende, favoreciendo la construcción del conocimiento de forma autónoma (Hernández, 2008).

El principal objetivo de la educación es que las personas aprendan. 
Para potenciar y maximizar ese aprendizaje, la mayoría del profesorado trata de personalizar en la medida de lo posible la enseñanza, lo que conlleva identificar las necesidades y capacidades individuales de discentes, desarrollando y programando contenidos flexibles de acuerdo a los ritmos de aprendizaje. Las clases tradicionales en numerosas ocasiones, no consiguen alcanzar esa diferenciación propia de un currículo adaptado a la diversidad. Algunos docentes optan por una estrategia semipresencial, ayudados de las TIC (Davies, Dean, \& Ball, 2013).

Un modelo que proporciona flexibilidad, adaptación a ritmos de aprendizajes, autorregulación, facilidad de trabajo colaborativo y cooperativo, apoyado en las TIC, entre otras cualidades, y por ello se ha seleccionado en nuestra propuesta didáctica, sería el que se conoce como flipped classroom o aula invertida.

El aprendizaje bajo el modelo flipped classroom, permite a los docentes atender con mayor profundidad a todo el alumnado. Este enfoque invierte el modelo tradicional de aula al introducir los conceptos del curso antes de la clase, lo que permite a educadores emplear el tiempo de clase para atender y guiar a cada estudiante a través de aplicaciones activas, prácticas innovadoras de los contenidos esenciales de la materia (Santiago \& Bergmann, 2018).

Esto es solo un punto de partida para comenzar, dentro de un marco de investigación-acción llevado a cabo durante dos cursos, una metodologia en el aula basada en el flipped classroom. El desarrollo de la misma puede ser tan heterogéneo como diferente sea el profesorado y los grupos de clase.

El objetivo de este trabajo es, mediante la observación, planificación, experimentación y reflexión, propias de la investigación-acción, obtener información que evidencie que utilizando el modelo flipped classroom se consigue fomentar la autorregulación y metacognición en el proceso de enseñanza-aprendizaje de la Didáctica de la Estadística.

\section{Marco conceptual de la propuesta}

Los antecedentes teóricos de esta investigación-acción, están en el modelo flipped classroom, un modelo que fomenta la autorregulación del aprendizaje, favoreciendo la metacognición, entre otros aspectos. Este marco se presenta como un contexto óptimo para desarrollar una propuesta didáctica innovadora en $4^{\circ}$ curso del Grado en Educación Primaria, con los contenidos propios de la Didáctica de la Estadística para maestras y maestros. En este caso, las docentes son al mismo tiempo investigadoras, analizando su propia práctica para mejorarla, 
siendo un proceso dinámico, y permitiendo al alumnado participar y responsabilizarse de su propio aprendizaje.

\section{Aprendizaje autorregulado y metacognición}

Llorente (2013) citando a Zimmerman (2000), define el aprendizaje autorregulado como "la capacidad de un estudiante para participar independientemente y proactivamente en los procesos de automotivación y conducta que ayuden a incrementar el logro de sus propias metas de aprendizaje" (p.70). El autor añade además esta definición, "E1 aprendizaje autorregulado puede ser considerado como una habilidad, donde los estudiantes deben saber cómo establecer metas, lo que se necesita para alcanzar dichos objetivos y la forma de alcanzar estos objetivos en la realidad" (Llorente, 2013, p.70).

El aprendizaje autorregulado se puede estructurar en tres fases cíclicas, una fase inicial en la que se establecen objetivos de aprendizajes y el interés por dichos objetivos, una segunda fase, selección y realización de tareas de aprendizaje, y seguimiento del proceso de aprendizaje. Y la última fase de autorreflexión, autocontrol de resultados, y toma de decisiones para reiniciar el proceso, hasta alcanzar el objetivo (Llorente, 2013).

En numerosos artículos científicos sobre los procesos de enseñanza y aprendizaje, aparecen términos y expresiones como "aprender a aprender", "aprendizaje para toda la vida", "autorregulación", "aprendizaje autodirigido", o incluso con anglicismos como "lifelong learning", "self-regulated learning". Más allá de las propias expresiones, es evidente que en una sociedad donde la información y el conocimiento son un bien y una necesidad, es esencial por parte del profesorado el desarrollar en su alumnado capacidades para gestionar esa información y convertirla en conocimiento adaptado a la diversidad y necesidades de cada uno. Para crear aprendices permanentes, que sepan enfrentarse a los cambios e incertidumbres de nuestro tiempo, es preciso enseñarles a aprender, a planificar, autorregular y autoevaluar sus propios procesos de aprendizaje (Coll \& Monereo, 2008).

Los mismos autores, afirman que el uso de las TIC requiere una determinada ordenación y visibilidad de las acciones, se puede ofrecer una rápida respuesta a dichas acciones, lo que favorece la toma de conciencia y la autorregulación cognitiva, procesos propios para construir el conocimiento condicional (en qué condiciones hacer qué) (Coll \& Monereo, 2008). Además, las TIC promueven una interacción dinámica con distintos objetos de conocimientos, realizando un intercambio de información entre lo que producimos y lo que otros hacen. Las distintas formas en las que se presenta la información y la 
facilidad para crear y modificar redes de conocimiento, favorecen las posibilidades de aprender nuevas formas de gestión de conocimiento.

Al igual que ocurre con estudiantes a distancia, cuando un estudiante se enfrenta al aprendizaje de manera más autónoma, debe tomar decisiones en cuanto a la organización del entorno de aprendizaje, la gestión del tiempo, la interacción con el material didáctico (Coll \& Monereo, 2008). El estudiante debe marcar una estrategia, en la que incluyen dimensiones propias de la autorregulación del aprendizaje, la planificación, supervisión y evaluación de un plan de aprendizaje o cómo hacer frente a las posibles dificultades.

Sin embargo, a diferencia de la educación a distancia, con el modelo flipped classroom, el estudiante no debe sentir esa sensación de estar solo, de no tener el apoyo del profesorado o sus iguales, ya que solo durante una parte del proceso de aprendizaje se enfrenta de manera individual al mismo.

Los discentes deben adquirir conocimientos y habilidades necesarias para hacer frente a sus proyectos de aprendizaje, de ahí el concepto de autorregulación del aprendizaje, y en esto debe enfocarse parte de la tarea del docente, en saber transmitir esas habilidades a su alumnado. Mediante este modelo, autores como Chen, L., Chen, T.L., y Chen, N.S. (2015) y Sánchez-Cruzado, Sánchez-Compaña y Ruiz (2019), afirman que la autonomía, la propia motivación y la iniciativa personal mejoran y se fortalecen, lo que hará que desarrollen habilidades y competencias para la autorregulación del aprendizaje a lo largo de sus vidas. Investigaciones como las de Del Arco, Flores y Silva (2019), llegan a la conclusión de que, con el modelo flipped classroom, se consiguen más oportunidades para que el alumnado se involucre en su propio aprendizaje, siendo el centro del mismo, y se alcanza una participación activa tanto en su formación, como en su evaluación.

Por otro lado, y alcanzando un nivel superior en el proceso enseñanza-aprendizaje, Monereo y Barberá (2000) definen la metacognición como ese proceso relativo al conocimiento o conciencia que tienen las personas de su proceso de aprendizaje, qué saben y cómo están aprendiendo.

En la metacognición se pueden destacar tres aspectos básicos, el conocimiento y conciencia de sí mismo y de los propósitos de aprendizaje, conocimiento sobre estrategias para resolver situaciones propuestas, y la autorregulación (Brown \& Baker, 1984).

La autorregulación implica entonces un conocimiento propio, y del proceso de aprendizaje personal. Es decir, el discente selecciona de forma consciente su estrategia de aprendizaje, y de forma autónoma, es capaz de transferir procesos y habilidades a otros contextos o situaciones que tengan elementos comunes. 
El alumnado que alcanza la consciencia de sus habilidades metacognitivas, tiene mayor capacidad de aprendizaje, consiguiendo mayor eficiencia en el mismo, con lo que obtiene resultados académicos más favorables. El entorno virtual, amplía las posibilidades de adaptación personalizada a los distintos ritmos de aprendizaje, permitiendo diseñar un compendio de tareas apropiadas al nivel de conocimiento, hasta llegar a la metacognición, que facilita el control y monitorización para una adecuada autorregulación (Osses \& Jaramillo, 2008).

\section{La educación matemática y la educación estadistica}

La educación matemática, debe ir más allá del aprendizaje del uso de herramientas e instrumentos puramente matemáticos, de hecho, se debería invertir esa tendencia, para dedicar más atención a las necesidades formativas de las personas, es decir a desarrollar capacidades y competencias matemáticas, saber tratar de forma crítica la información, también fomentar su autonomía, el espíritu crítico y la confianza en el propio pensamiento (Gonzalez-Mari, 2020). Se debe conseguir que la formación en general, proporcione al alumnado un cierto grado de autonomía física, personal, social y moral (López-Melero, Mancila, \& Sole, 2016). Sería necesario alcanzar por un lado una formación que permita al alumnado comprender las matemáticas para adaptarse al medio, organizarlo y transformarlo, y por otro lado prepare al individuo para analizar todas las opciones presentes en una situación y seleccionar las mejores (Macías-García et al., 2018).

De entre los objetivos más importantes de la educación, y en concreto de la educación matemática está conseguir que el alumnado desarrolle su capacidad de pensamiento, aprender a pensar. Educar con las matemáticas, se convierte en fomentar el desarrollo del pensamiento matemático (Lorenzato, 2015).

La Matemática es una materia interdisciplinar, relacionada con casi todos los campos de la realidad, como son las ciencias sociales, los juegos, la música o la política, no solo y exclusivamente con el ámbito científico-tecnológico, tiene a su vez un triple carácter, instrumental, formativo y funcional (Gonzalez-Mari, 2020; Sánchez-Compaña, GarcíaRuíz, \& Sánchez-Cruzado, 2019). Existen determinadas actividades matemáticas y científicas que potencian la adquisición de capacidades cognitivas. Se podría afirmar que todos los currículos de matemáticas y ciencias, contienen y resaltan la enseñanza de las matemáticas, por su valor formativo, no solo en el aspecto cognitivo, también por su relación con el campo afectivo (Rico, 1990).

Rico, Flores y Ruiz-Hidalgo (2015), actualizan esta afirmación, y 
establecen la necesidad de educar en matemáticas, considerando que el conocimiento matemático ha de plantear y responder cuestiones reales, y resolver problemas en diferentes contextos.

Las matemáticas son imprescindibles en la formación básica de las personas. La educación matemática, se trata de una actividad social clave para la formación de ciudadanas y ciudadanos en una sociedad democrática avanzada (Rico \& Sierra, 2000). Conseguir una ciudadanía responsable socialmente, suficientemente autónoma debe ser una de las tareas de la educación, en concreto en la educación matemática que es la que nos compete en este caso.

Dentro de la educación matemática, cabe distinguir la educación estadística.

Durante las últimas décadas, la estadística ha alcanzado mayor relevancia y reconocimiento que otras áreas de la matemática. Se presenta como ese conjunto de herramientas que ayudan a argumentar basados en la evidencia, que además permiten discutir afirmaciones basadas en datos. Esto es una habilidad necesaria en la ciudadanía, ya que la correcta presentación de datos es imprescindible para sumar credibilidad a cualquier información, investigación o noticia (MolinaPortillo et al., 2018).

Para definir la estadística, es imprescindible citar a Moore (1991), quien habla de la estadística como la ciencia de los datos. A partir de datos empíricos, se consigue mejorar la compresión de los hechos, siendo muy importante el contexto. El alumnado no está habituado a trabajar con datos de situaciones reales, que requieren de una interpretación profunda (Sánchez \& Batanero, 2011).

Burril y Biehler (2011), hablan de cultura estadística, para referirse a la compresión de las ideas estadísticas fundamentales. Dichas ideas, pueden ser adaptas a distintos niveles educativos, entrando en menor o mayor profundidad en función del curso.

La cultura estadística involucra a dos competencias relacionadas, por un lado, la capacidad para descifrar y valorar críticamente la información estadística, datos o fenómenos que pueden aparecer en distintas situaciones o contextos. Y, por otro lado, la capacidad para discutir o comunicar argumentos respecto a la información estadística (Gal, 2002).

Watson (2006), en la misma línea, plantea tres elementos fundamentales en la cultura estadística, el desarrollo del conocimiento básico de conceptos estadísticos y probabilísticos, también el conocimiento de razonamientos y argumentación estadística al presentarse en un contexto concreto. Y finalmente, avanza un paso más añadiendo una actitud crítica capaz de cuestionar argumentos, basados en la evidencia estadística. Este tercer punto, enlazaria con la tercera 
fase de la autorregulación de la que habla Llorente (2013), en la que se llega a un proceso de autorreflexión, autocontrol de resultados, y toma de decisiones.

Dentro de la estadística, cabe destacar la representación gráfica de los datos, y su aprendizaje, tanto la elaboración como la interpretación son fundamentales. Este hecho es clave, debido a la fuerte presencia en los medios de comunicación e Internet de gráficos estadísticos. Realizar e interpretar tablas y gráficos, son instrumentos imprescindibles en el razonamiento estadístico (Wild y Pfannkuch, 1999).

La interpretación crítica de datos estadísticos, la reflexión y análisis de resultados, la capacidad de tomar decisiones en función de dicho análisis, son procesos fundamentales, para fomentar la autonomía y el espíritu crítico de la futura ciudadanía. Teniendo en cuenta, que se está hablando de una formación de futuros maestros y maestras, es su responsabilidad, no solo desarrollar esa autonomía, sino que además debe potenciarla en su futuro alumnado.

\section{Flipped classroom en Didáctica de la Estadistica}

De partida, el modelo flipped classroom, da prioridad al autoaprendizaje, a la reflexión y fomenta una construcción activa del conocimiento (Andia, Santiago, \& Sota, 2020).

Según Tourón y Santiago (2015), un modelo educativo basado en flipped classroom aporta beneficios entre los que se destacan:

- Permite atender las diferencias individuales durante mayor tiempo.

- El profesorado tiene la oportunidad de compartir información y conocimientos entre iguales, con el alumnado, con las familias y el resto de la comunidad educativa.

- El alumnado dispone de la posibilidad de acceder tantas veces como necesite a los contenidos facilitados por su profesorado.

- Aumenta las posibilidades de aprendizaje colaborativo.

- Favorece la autonomía y autorregulación del aprendizaje.

Esta lista de beneficios es compartida también por otros autores y autoras, que muestran evidencias en sus trabajos de investigación (Del Arco et al. (2019); Sánchez-Cruzado et al. (2019)).

Cabría pensar que el flipped classroom podría ser solo una preparación o lectura previa de un material didáctico presentado por el profesorado antes de una clase, en la que se propondrán tareas más reflexivas, colaborativas y participativas. Sin embargo, con ayuda de las TIC, se favorece el acceso a contenidos enriquecidos, en un formato atractivo, en cualquier lugar y a cualquier hora (Davies et al., 2013).

"Invertir" una clase es mucho más que la edición y distribución de un vídeo o de cualquier otro tipo de contenidos multimedia. 
Se trata de un enfoque integral que combina la instrucción directa con métodos constructivistas, actuaciones de compromiso e implicación de los estudiantes con el contenido del curso y la mejora de su comprensión conceptual (Tourón \& Santiago, 2015, p.209).

Para invertir una clase tradicional se pueden utilizar distintos recursos y estrategias, tantas como ideas pueda tener el profesorado que decida abordar este tipo de retos. Una de las posibilidades es elaborar pequeños vídeos y vodcast grabados por el propio docente, o utilizar otros vídeos ya existentes en la red Internet con los conceptos y/o explicaciones necesarias. Cualquier formato seleccionado debe incluir contenidos claves en el aprendizaje y serán visualizados como tareas en casa (Abeysekera \& Dawson, 2015).

Por otro lado, Rotellar y Cain (2016) proponen una serie de premisas como recomendaciones de partida a la hora de implantar y desarrollar una estrategia metodológica basada en la clase invertida:

1. Usar la programación académica para determinar qué contenidos debe presentarse fuera del aula y cómo diseñar las actividades de aprendizaje en el aula.

2. Facilitar posibilidades de desarrollo académico individuales y en grupo para ayudar a los miembros universitarios al cambio de paradigma.

3. Reconocer que la dinámica natural de la clase, puede requerir la necesidad de apoyo en el aula.

4. Ser conscientes de que el cambio de reglas puede abrumar a una gran cantidad de estudiantes que deben ser re-educados en cómo tener éxito en esta nueva aula.

5. Conectar correctamente las actividades previas a la clase, con las actividades que se realizarán en el aula.

6. Asesorar al alumnado para que entiendan exactamente qué necesitan saber o hacer antes de ir a clase.

7. Dar opciones a estudiantes para preguntar y/o aclarar la información de los contenidos propuestos y expuestos por el profesorado fuera del aula.

8. Focalizar las actividades en clase para que ayude a los discentes a pensar como personas expertas.

9. Fomentar oportunidades de evaluación formativa para entender qué saben y qué no saben.

10. Resistirse al impulso de enseñar de nuevo al alumnado que no se prepara correctamente. Se les debe hacer conscientes de su responsabilidad como futuros profesionales.

11.Tener cuidado de no sobrecargar a estudiantes con demasiado contenido fuera de clase, demasiados vídeos, materiales, lecturas 
y otros documentos o recursos.

12. Valorar qué conocimientos, habilidades, comportamientos y actitudes se desean del alumnado. No sirve de nada enseñar y desarrollar en el aula el pensamiento crítico y la resolución de problemas y evaluar solo conocimientos.

13. Estar preparados para adaptar las actividades de enseñanza y aprendizaje según se observen cómo contribuyen o no al aprendizaje.

Cuando un docente decide invertir sus clases, es decir poner en marcha el modelo flipped classroom, se le recomienda que las primeras clases sean de adaptación a esta nueva forma de trabajar. Se debe detallar cómo va a transcurrir el tiempo en el aula durante el curso, qué tipo de trabajo se va a realizar, tanto dentro como fuera del aula, y transmitir la necesidad de la implicación y adquisición de nuevas responsabilidades por parte del alumnado.

Invirtiendo la clase, se dispone de más tiempo en el aula para trabajar aprendizajes más complejos, de mayor nivel cognitivo, incluida la metacognición, y trabajando de forma autónoma enseñanzas más sencillas (Santiago, 2019).

Kuiper et al. (2015) llevaron a cabo una experiencia en el aula implementando flipped classroom en clase de estadistica, y comprobaron que utilizando vídeos para mostrar conceptos estadísticos en distintos contextos reales, su alumnado parecía estar más convencido de la importancia de la estadistica en otras disciplinas. Touchton (2015), en su experiencia trabajando Estadistica Avanzada mediante el modelo flipped classroom, apreció que su alumnado, mejoró en áreas de mayor dificultad, que pudieron profundizar en clase, teniendo el alumnado la percepción de haber aprendido más.

\section{Metodología}

Cuando se pretende llevar a cabo una investigación en el ámbito educativo, en la que se trabaja con grupos relativamente reducidos, donde además el profesorado toma el rol de docente-investigador, una metodología comúnmente utilizada es la investigación-acción.

La investigación-acción en el ámbito de la didáctica, pretende reflexionar sobre una práctica educativa, con el objetivo de mejorar la calidad formativa (McNiff, 2003). Es un proceso cíclico de observación, y diagnóstico, planificación, acción, de nuevo observación y reflexión sobre resultados, tratando de producir cambios que mejoren el proceso enseñanza-aprendizaje (Bausela, 2004).

Esta experiencia se llevó a cabo tras comprobar en la primera fase 
de observación, durante varios cursos previos al 2017-2018, en la asignatura Didáctica de la Medida, concretamente en el bloque de Estadística y Probabilidad, que se debian emplear numerosas sesiones para trabajar adecuadamente este bloque. La mayoria del tiempo invertido se dedicaba a contenidos puramente teóricos, sin llegar a profundizar en actividades prácticas en las que se fomentara ese tercer nivel de análisis crítico de los resultados, propios de la metacognición.

En una segunda fase de planificación, se organiza la nueva dinámica de clase. Concretamente se seleccionan los contenidos puramente instrumentales y trasladándolos "fuera del aula", se deja para trabajar dentro del aula el aspecto más funcional y formativo de la estadística. Es decir, permite emplear el tiempo en el aula para, además de interpretar y evaluar la información estadística, desarrollar la capacidad de discutir y comunicar opiniones. Disponiendo de más tiempo en el aula, para trabajar la estadística de forma práctica y contextualizada, se produce un conocimiento más profundo de conceptos estadísticos básicos, propiciando en el alumnado una actitud crítica.

En la tercera fase de acción, de la investigación-acción, se lleva a cabo una experiencia innovadora, basada en el modelo flipped classroom, que recoge las necesidades de esta propuesta. Del desarrollo de la propuesta, se obtendrían evidencias, tras un profundo proceso reflexivo y de evaluación, correspondiente a la siguiente fase de la investigación-acción.

Con las evidencias encontradas, se planifica el siguiente curso 20182019 , continuando un nuevo ciclo en la investigación-acción, en el que se realizaron varias mejoras, como la mejor edición de vídeos, pero se desarrolló el mismo modelo en el aula.

Se debe informar al alumnado participante, de cómo se va a desarrollar el nuevo curso, con un planteamiento diferente a la metodologia tradicional. Hay que conseguir lo antes posible tener a unos discentes informados y que se hagan responsables de su aprendizaje. Esta es una de las características de este modelo, fomentar la autonomía y responsabilidad del alumnado.

Una vez se desarrolla la experiencia, se trata de comprobar si realmente el modelo flipped classroom, propicia el desarrollo de actividades que favorezcan la autorregulación del aprendizaje, alcanzando niveles reales de metacognición.

\section{Población y muestra}

La población participante fue el alumnado de la Facultad de Ciencias de la Educación de la Universidad de Málaga en el curso 2017-2018 y 2018-2019. En este caso se ha optado por un muestreo incidental o 
casual, seleccionando los individuos a los que se tiene fácil acceso. La muestra pertenece a las asignaturas de Didáctica de la Medida de los grupos $\mathrm{A}$ y C de $4^{\circ}$ curso, 308 personas, 147 alumnas y alumnos en 2017-2018 y 161 en el curso 2018-2019.

\section{Desarrollo de la experiencia}

Siguiendo las premisas de Rotellar y Cain (2016) sobre el modelo flipped classroom, mencionadas en el punto 2.3 de este artículo, este fue el desarrollo que tuvo esta experiencia:

Respecto al punto primero, se seleccionan varios bloques, de entre los contenidos recogidos en la guía docente de la asignatura de Didáctica de la Medida. Entre ellos, "Medidas relacionadas con el análisis de datos y el pensamiento estadístico elemental" y "Didáctica de la medida de la incertidumbre, el azar y la probabilidad". Se seleccionan intencionadamente los bloques con contenido estadístico para implantar con la metodología flipped classroom, ya que de acuerdo con Chen et al. (2015), los contenidos estadísticos, se prestan al trabajo en grupo y aprendizaje colaborativo. Una vez seleccionados estos contenidos, se fijan los objetivos de aprendizaje.

Sobre la actividad individual y grupal de la segunda premisa: en el aula, como punto de partida y actividad del grupo clase, se genera un debate sobre la importancia de la educación estadística, errores, dificultades y obstáculos en su aprendizaje, vivencias previas en las etapas de educación primaria, secundaria y bachillerato. Se propone como trabajo de grupos reducidos, realizar dos estudios estadísticos de variables aleatorias cualitativas y cuantitativas, que sean de su interés. Se pretende, por un lado, aprender de forma práctica las nociones de la estadística, y por otro, conocer en profundidad al grupo clase. De las múltiples variables que se barajan, "edad", "número de personas con las que conviven en casa", "libros leídos en el último año", "tipo de música", entre otros, merece la pena destacar aquellas variables que ponen de manifiesto el cambio cultural, social y de costumbres, de la juventud actual, "tiempo medio que emplean al dia utilizando redes sociales", "número de tatuajes", "número de países a los que han viajado", "participaciones en ONG", "tipos de ONG", "países a los que ha viajado", "fotos y videos subidos a Instagram al dia", entre otros.

Una vez diseñado el cuestionario, de forma conjunta y consensuada, a partir del cual obtener variables para los análisis estadísticos, se elabora una tabla para la recogida de datos. Como actividad individual se propone traer las respuestas a dicho cuestionario, para poner en común los datos, repartirlos y realizar su posterior análisis en grupos dentro del aula. 
Se prepararon vídeos con contenidos puramente teóricos para ver fuera del aula (uno de ellos puede verse en Sanchez-Compaña et al. (2019), como trabajo individual. A partir de estos vídeos el alumnado disponía de la información suficiente para el posterior análisis estadístico en el aula, como trabajo grupal. Se realiza la adaptación oportuna de contenidos, tal y como indica la premisa trece de Rotellar y Cain (2016). El alumnado tiene en el aula oportunidad de preguntar sobre los contenidos que se han presentado en los vídeos en todo momento. El análisis realizado en el aula en las siguientes sesiones, los hará razonar y argumentar como futuras personas expertas en estadística. Esto responde a las premisas séptimas y octavas.

Es evidente que las actividades dentro y fuera del aula, estuvieron intimamente relacionadas como indica la premisa quinta.

Relativo a la necesidad de un guía en el aula, premisa tercera de Rotellar y Cain (2016), las docentes que participaron de la experiencia, asumieron el rol de guía y apoyo. Se trató de mantener el control del aula, reconduciendo el aprendizaje en cualquier momento, profundizando en puntos de la materia que, con un planteamiento de clase tradicional, sería prácticamente imposible. El papel del docente es imprescindible, de acuerdo también con la premisa en la que se destaca su función de asesorar al alumnado para que tenga claro qué deben saber o hacer.

En la premisa cuatro, se debe concienciar al alumnado del cambio de reglas. Se cuenta con un alumnado acostumbrado a permanecer en el aula como mero espectador, y ahora tiene que ser un agente activo, requiriendo un mayor esfuerzo por su parte.

El alumnado asume, en relativamente poco tiempo, su rol de personaje activo en el aula. Puede observar cómo las respuestas a sus dudas y reflexiones llegan en la misma aula, sin dilatarse en el tiempo, como consecuencia de trabajos de investigación de grupo, o mediante resolución de problemas, de la mano de su profesora y de sus propios compañeros y compañeras.

En la propia experiencia llevada a cabo, ocurrió que algunos alumnos y alumnas, no vieron los primeros vídeos con contenidos teóricos, perdiendo los primeros días el ritmo en las sesiones iniciales en el aula. De acuerdo con la premisa décima de Rotellar y Cain (2016), no se deben repetir las explicaciones en el aula, solo invitar a ese alumnado a ver los vídeos durante el tiempo en clase, en detrimento del tiempo disponible para trabajar en actividades de nivel superior de aprendizaje. De esta forma, se consiguió fomentar la responsabilidad del alumnado.

Una vez finalizado el desarrollo de las tareas grupales, relacionadas con el análisis estadístico, se lleva a cabo una puesta en común y debate 
posterior sobre los resultados que se obtienen. De esta forma, se desarrollaron habilidades de razonamiento y discusión de resultados, y una actitud critica en situaciones realmente contextualizadas.

Y con todo el proceso concluido, las docentes-investigadoras, entran en la fase de evaluación y reflexión de los resultados en la investigaciónacción. Pueden observar qué tipo de aprendizajes se han alcanzado, qué mejoras se han producido, o qué debilidades se deben superar para la siguiente fase y nueva planificación del siguiente curso. En el siguiente curso, se repitió la experiencia.

\section{Técnicas para la recogida de información}

En esta investigación-acción, con un claro enfoque cualitativo, para obtener información del proceso enseñanza-aprendizaje, se utiliza la observación participante y el análisis de documentos (diario docente, actividades y trabajos realizados por el alumnado, grabaciones de debates), tomando evidencias de las mejoras producidas respecto a años anteriores. Para poder hablar de fiabilidad en esta investigación, se ha llevado a cabo el proceso durante dos cursos, utilizando las mismas técnicas de recogida de información, comprobando que se obtienen resultados muy similares.

Además, las docentes-investigadoras, que han desarrollado la práctica durante los dos cursos, utilizaron el diario de clase como instrumento que permitia contrastar reflexiones para garantizar también la validez de la investigación. Esta experiencia es fácilmente replicable, como se ha podido comprobar.

\section{Resultados}

Tras analizar los diarios de las docentes-investigadoras, las grabaciones de debates, y los distintos documentos generados por el alumnado, se comprueba, que hay diferencias en las formas de trabajar $\mathrm{y}$ aprender con y sin flipped classroom. En la Tabla 1, se muestran algunas de esas evidencias.

Tabla 1.

Algunos resultados de la observación inicial y de la evaluación de la acción en los dos cursos

\begin{tabular}{lll}
\hline $\begin{array}{l}\text { Variable a } \\
\text { estudio }\end{array}$ & $\begin{array}{l}\text { De la observación inicial, sin } \\
\text { flipped classroom }\end{array}$ & $\begin{array}{l}\text { De la evaluación y reflexión de la } \\
\text { acción, con flipped classroom } \\
\text { (cursos 2017-2018 y 2018-2019) }\end{array}$ \\
\hline
\end{tabular}


El modelo flipped classroom, una forma de promover la autorregulación y la metacognición en el desarrollo de la educación estadística

\begin{tabular}{|c|c|c|}
\hline $\begin{array}{l}\mathrm{N}^{\circ} \text { sesiones } \\
\text { destinada a } \\
\text { D. Estadistica }\end{array}$ & $\begin{array}{l}5 \text { sesiones: a) } 4 \text { de clases } \\
\text { magistrales con contenidos } \\
\text { teóricos fundamentalmente, b) } 1 \\
\text { de actividades de aplicación }\end{array}$ & $\begin{array}{l}3 \text { sesiones: a) debate inicial y } \\
\text { definición del problema, b) actividades } \\
\text { en el aula de mayor nivel cognitivo, c) } \\
\text { exposición de resultados y debate } \\
\text { final }\end{array}$ \\
\hline $\begin{array}{l}\text { Tipo de } \\
\text { actividades } \\
\text { en el aula }\end{array}$ & $\begin{array}{l}\text { Instrumentales individuales, } \\
\text { alumnado pasivo }\end{array}$ & $\begin{array}{l}\text { Trabajo grupal, resolución de } \\
\text { problemas, reflexión, análisis, } \\
\text { actividades de mayor nivel cognitivo, } \\
\text { decisiones sobre propio aprendizaje }\end{array}$ \\
\hline $\begin{array}{l}\text { Tipo de } \\
\text { actividades } \\
\text { fuera del aula }\end{array}$ & $\begin{array}{l}\text { Lecturas, resolución de } \\
\text { problemas de forma individual }\end{array}$ & Visionado de vídeos, lecturas \\
\hline $\begin{array}{l}\text { Tipo de } \\
\text { evaluación }\end{array}$ & $\begin{array}{l}\text { Pregunta en examen, actividad } \\
\text { instrumental }\end{array}$ & $\begin{array}{l}\text { Valoración del trabajo grupal llevado } \\
\text { a cabo en sesiones de clase y de las } \\
\text { exposiciones finales }\end{array}$ \\
\hline Resultados & $\begin{array}{l}\text { Conocimiento y comprensión } \\
\text { procedimental, aplicación } \\
\text { instrumental }\end{array}$ & $\begin{array}{l}\text { Conocimiento y comprensión } \\
\text { procedimental, aplicación } \\
\text { instrumental. Autorregulación, } \\
\text { autonomia, metacognición, espíritu } \\
\text { crítico }\end{array}$ \\
\hline
\end{tabular}

Durante las sesiones en el aula, se pudo comprobar que no solo se realizaban, actividades estadísticas puramente instrumentales. Se consiguieron desarrollar tareas de análisis de datos estadísticos y argumentación de resultados contextualizados en una serie de variables que fueron elegidas por el alumnado de acuerdo a sus inquietudes de forma autónoma. Además, durante el debate final, se consiguió cuestionar de forma crítica los resultados obtenidos. También hay que destacar que, durante el resto de sesiones, se producían pequeños debates de forma espontánea, discutiendo los distintos aprendizajes producidos. Los debates eran grabados, y han sido fuente de información.

En la sesión de debate final en las que se comparten los resultados, se observaron datos curiosos, que fomentaron la reflexión sobre el cambio social. Destacando uno de los casos, en el curso 2018-2019, respecto a la variable cuantitativa "número de libros leídos al año", la moda era 0 y la media 2.3 libros, es decir la mayoría del alumnado no había leído ningún libro en un año. Ante ese asombroso dato, sobre todo considerando que los participantes serán futuros docentes, la profesora inicia un debate para realizar una valoración crítica de este hecho. Entre otras tareas como docentes, una de ellas será el fomento a la lectura en su alumnado en un futuro. El grupo asume la crítica y realiza una reflexión en profundidad.

Al analizar otra de las variables cuantitativas, "número de países a los que han viajado", se obtiene una media de 7.2 paises, y una moda de 4. Otro hecho que llama la atención positivamente de la profesora, y da lugar a una reflexión complementaria a la anterior. 
Se llega a una valoración global sobre el grupo, el alumnado considera que hoy en día la cultura no les llega solo en un formato de libro, tiene otras formas de culturización más activas, como son experiencias vitales, que ocurren visitando otros países, culturas y tradiciones. Llegan incluso a afirmar que "hoy en dia somos cultas y cultos de otra manera".

Estas y otras reflexiones, favorecieron por un lado conocer al grupo con mayor profundidad, y por otro, para razonar y analizar el porqué de los distintos resultados. Observaron la funcionalidad de la estadística, contextualizada en sus propias experiencias personales. Obtuvieron evidencias estadísticas para poder de forma crítica, argumentar el perfil de una nueva generación de forma reflexiva y meditada (Watson, 2006). Es un proceso propio de la autorregulación del aprendizaje (Llorente, 2013).

\section{Conclusiones}

Con este trabajo, convencidas de que el modelo flipped classroom, se ajusta en gran medida a los deseos de una enseñanza más activa, participativa, colaborativa, que prepara a las personas para un mañana impreciso e indefinido, que fomenta la autonomía y la autorregulación del aprendizaje, se pretende aportar un pequeño grano de arena a los trabajos que confirman las mejoras, que este modelo produce en los procesos de enseñanza-aprendizaje. Concretamente y de acuerdo con Del Arco, Flores y Silva (2019), la autonomía es un elemento clave que se enriquece con este modelo.

La forma en la que se desarrollaron las sesiones, subrayan argumentos suficientes para continuar profundizando en este modelo, no exclusivamente en estadística, además en el resto de bloques de contenidos matemáticos de esta, y otras asignaturas del área de la Didáctica de la Matemática.

Ante este escenario, no cabe duda, de que realmente se está produciendo un proceso de aprendizaje, no solo de conocimientos.

El alumnado parece entender que la matemática, en concreto la estadística, les ha servido para concebir el medio, organizarlo y transformarlo en información contrastada.

Tomar la responsabilidad del proceso de aprendizaje, no es un asunto banal, y la mayoria de los estudiantes, en este caso universitarios, deben ser conscientes y estar dispuestos a experimentar, y comprobar que la propia metodologia de aprendizaje es a su vez aprendizaje activo. No solo se está adquiriendo conocimiento, además, se están adquiriendo otras destrezas, habilidades y competencias a través del modelo de enseñanza-aprendizaje. El aprender a aprender, 
aprender a pensar, reflexionar y tener una actitud critica, entre otros, son fundamentales para desarrollar competencias propias de la cultura estadística, y con ella de la educación matemática. Se están desarrollando otras habilidades, características de la autorregulación y metacognición. El alumnado, está siendo capaz de razonar, de entender qué está aprendiendo, cómo y para qué, desarrollando ese espíritu crítico tan necesario y deseable socialmente.

\section{Referencias}

Abeysekera, L., \& Dawson, P. (2015). Motivation and cognitive load in the flipped classroom: definition, rationale and a call for research. Higher Education Research \& Development, 34(1), 114. https://doi.org/10.1080/07294360.2014.934336.

Andía Celaya, L. A., Santiago Campión, R., \& Sota Eguizabal, J. M. (2020). ¿Estamos técnicamente preparados para el flipped classroom? Un análisis de las competencias digitales de los profesores en España. Contextos Educativos. Revista de Educación, (25), 275-311.

Baker, L., \& Brown, A. (1984). Metacognitive skills and reading. En Pearson, P.D Handbook of reading research (pp. 353-396) EE.UU: Longman.

Bausela Herreras, E. (2004). La docencia a través de la investigaciónacción. Revista Iberoamericana de Educación, 35(1), 1-9.

Bishop, A. J. (1999). Enculturación Matemática. La Educación Matemática desde una perspectiva cultural. Editorial PAIDOS.

Burrill, G., \& Biehler, R. (2011). Fundamental statistical ideas in the school curriculum and in training teachers. En C. Batanero, G. Burrill y C. Reading (Eds.), Teaching statistics in school mathematics. Challenges for teaching and teacher education - A joint ICMI/ IASE study (pp. 57-69). Dordrecht: Springer.

Chen, L., Chen, T.-L., \& Chen, N.-S. (2015). Students' Perspectives of Using Cooperative Learning in a Flipped Statistics Classroom. Australasian Journal of Educational Technology, 31(6), 621-640.

Coll, C., \& Monereo, C. (2008). Psicologia de la educación virtual. 
Ediciones Morata.

Davies, R. S., Dean, D. L., \& Ball, N. (2013). Flipping the classroom and instructional technology integration in a college-level information systems spreadsheet course. Educational Technology Research and Development, 61(4), 563-580. https: / /doi.org/ 10.1007/s1 1423-013-9305-6.

Del Arco Bravo, I., Flores Alarcia, O., \& Silva, P. (2019). El desarrollo del modelo flipped classroom en la universidad: impacto de su implementación desde la voz del estudiantado. Revista de Investigación Educativa, 37(2), 451-469. https://doi.org/10.6018/rie.37.2.327831 .

Gal, I. (2002). Adult's statistical literacy: Meaning, components, responsibilities. International Statistical Review 7O(1), 1-25.

González-Mari, J. L. (2020). Claves para una educación matemática humanista. Uno, 88, 49-59.

Hernández Requena, S. R. (Ed.). (2008). El modelo constructivista con las nuevas tecnologias, aplicado en el proceso de aprendizaje. Revista de Universidad y Sociedad del Conocimiento, RUSC, 5(2), 26-35.

Kuiper, S. R., Carver, R. H., Posner, M. A., \& Everson, M. G. (2015). Four Perspectives on Flipping the Statistics Classroom: Changing Pedagogy to Enhance Student-Centered Learning. PRIMUS, 25(8), 655-682. https: / /doi.org/ 10.1080/10511970.2015.1045573

Llorente Cejudo, M. (2013). Aprendizaje autorregulado y PLE. Edmetic, Revista de Educación Mediática y TIC, 2(1), 63-79.

López-Melero, M., Mancila, I., \& Sole, C. (2016). Escuela Pública y Proyecto Roma. Dadme una escuela y cambiaré el mundo. Revista Interuniversitaria de Formación del Profesorado, 30(1), 49-56.

Lorenzato, S. (2015). Para aprender matemáticas. Autores Associados (Editora Autores Associados LTDA)

Macías-García, J. A., Martín-Gámez, C., González, J. L. \& García, F. (2018). Teleological structure of scientific and mathematical 
education. En Conference proceedings. New perspectives in science education 7th edition (pp. 227-230). libreriauniversitaria.it Edizioni.

McNiff, J. (2003). Action Research: Principles and Practice. Editorial Routledge Taylor \& Francis Gruop.

Molina-Portillo, E., Contreras, J. M., Ruz, F. \& Contreras, J. (2018). Evaluación de la cultura estadística en futuros profesores de educación primaria: Interpretación y argumentación de gráficos estadísticos. En L. J. Rodríguez-Muñiz, L. Muñiz-Rodríguez, A. Aguilar-González, P. Alonso, F. J. García García \& A. Bruno (Eds.), Investigación en Educación Matemática XXII (pp. 348357). Gijón: SEIEM.

Moore, D. S. (1991). Teaching statistics as a respectable subject. En F. Gordon y S. Gordon (Eds.), Statistics for the twenty-first century (pp. 14-25). Mathematical Association of America.

Monereo, C., \& Barberá, E (2000). Diseño instruccional de las estrategias de aprendizaje en entornos educativos no-formales. Monereo et al. Estrategias de aprendizaje. Madrid, Visor/Ediciones de la Universitat Oberta de Catalunya.

Osses Bustingorry, S. \& Jaramillo Mora, S. (2008). Metacognición: un camino para aprender a aprender. Estudios pedagógicos (Valdivia), 34(1), 187-197.

Rico, L. (1990). "Diseño curricular en Educación Matemática: Una perspectiva actual.”. In Llinares S. \& Sánchez V. (Eds.), Teoría y práctica en Educación Matemática Didáctica. Editorial Alfar.

Rico, L., Flores, P. y Ruiz-Hidalgo, J. F. (2015). Enseñanza de las matemáticas con sentido. UNO Revista de Didáctica de las Matemáticas, (70), 48-54.

Rico, L. \& Sierra, M. (2000). Didáctica de la matemática e investigación. En J. Carrillo \& L.C. Contreras (Eds.), Matemática española en los albares del siglo XXI (pp. 77-131). Editorial Hergué.

Rotellar, C. \& Cain, J. (2016). Research, Perspectives, and Recommendations on Implementing the Flipped Classroom. American Journal of Pharmaceutical Education, 80(2), 34. 
https: / /doi.org/10.5688/ajpe80234.

Sánchez, E. \& Batanero, C. (2011). Manejo de la información. En E. Sánchez (Coord.), Enseñanza y aprendizaje de las matemáticas, Casos y perspectivas (pp. 64-92). México, D. F.: Secretaría de Educación Pública.

Sánchez-Compaña, M. T., García-Ruíz, C. R. \& Sánchez-Cruzado, C. (2019). La integración de pensamiento matemático y social. Una práctica en formación inicial del profesorado de educación secundaria. En A. Codina y M. F. Moreno (Eds.), Investigaciones en Pensamiento Numérico y Algebraico (pp. 179-195). Universidad de Almería.

Sanchez-Compaña, M. T, Macías-García, J.A., Sánchez-Cruzado, C. \& Duarte Tosso, I. (2019). Video de las medidas de dispersión de las variables cuantitativas continuas. En RIUMA https: / / hdl.handle.net/10630/18396.

Sánchez-Cruzado, C., Sánchez-Compaña, M. T., \& Ruiz, J. (2019). Experiencias reales de aula invertida como estrategia Metodológica en la Educación Universitaria española. Publicaciones, 49(2), 39-58. doi:10.30827/publicaciones.v49i2.8270.

Santiago Campión, R. (2019). Conectando el modelo Flipped Learning y la teoría de las Inteligencias Múltiples a la luz de la taxonomia de Bloom. Magister: Revista miscelánea de investigación, 31(2), 45-54.

Santiago Campión, R. \& Bergmann, J. (2018) Aprender al revés: flipped classroom y metodologías activas en el aula. Editorial Planeta-Paidós.

Shaughnessy, J. M. (2007). Research on statistics learning and reasoning. En F. Lester (Ed.), Second handbook of research on mathematics teaching and learning (pp. 957-1010). Greenwich, CT:Information Age y NCTM.

Tourón, J. \& Santiago, R. (2015). El modelo Flipped Learning y el desarrollo del talento en la escuela. Revista de Educación, 368, 196-231. https://doi.org/10.4438/1988-592X-RE-2015-368288 
Touchton, M. (2015). Flipping the Classroom and Student Performance in Advanced Statistics: Evidence from a QuasiExperiment. Journal of Political Science Education, 11(1), 28-44. https: / /doi.org/10.1080/15512169.2014.985105

Watson, J. M. (2006). Statistical literacy at school: growth and goals. Mahwah, NJ: Lawrence Erlbaum Associates.

Wild, C. y Pfannkuch, M. (1999). Statistical thinking in empirical enquiry. International Statistical Review, 67 (3), 221-248.

Zimmerman, B. J. (2000). Attaining self-regulation: A social cognitive perspective. En M. Boekaerts, P. R. Pintrich, \& M. Zeidner (Eds.), Handbook of self-regulation (pp. 13-39). San Diego, CA, US: Academic Press. 
